\title{
Sound Source Omnidirectional Positioning Calibration Method Based on Microphone Observation Angle
}

\author{
Xu Yang $\mathbb{D},^{1,2}$ Hongyan Xing $\mathbb{D}^{1,2}$ and Xinyuan Ji $\mathbb{D}^{1,2}$ \\ ${ }^{1}$ Collaborative Innovation Center on Forecast and Evaluation of Meteorological Disasters, Nanjing University of Information Science \\ \& Technology, Nanjing 210044, China \\ ${ }^{2}$ Jiangsu Key Laboratory of Meteorological Observation and Information Processing, Nanjing University of Information Science \\ \& Technology, Nanjing 210044, China
}

Correspondence should be addressed to Hongyan Xing; xinghy@nuist.edu.cn

Received 15 May 2018; Accepted 9 August 2018; Published 4 October 2018

Academic Editor: Junpei Zhong

Copyright (c) 2018 Xu Yang et al. This is an open access article distributed under the Creative Commons Attribution License, which permits unrestricted use, distribution, and reproduction in any medium, provided the original work is properly cited.

To get source azimuth from microphone observation angle of view in a complex real environment, this article, on the basis of the analysis of geometric positioning method, established a seven-element microphone array model and proposed a sound source omnidirectional positioning calibration method based on microphone observation angle. By using a seven-element array to invert the position and angle of a sound source, the relative time delay value of a pair of microphones on the vertical axis of the coordinate system is used to determine the elevation angle polarity and realize the omnidirectional sound source positioning. The array parameters, sound velocity, array size, horizontal deflection angle, elevation angle, and sound source are analyzed, and the error method is proposed. The sound source data was measured using the microphone array perspective, and a new Cartesian coordinate system was established based on the observation angle of view for omnidirectional positioning calibration of the sound source. The simulation results show that the position error of the method is about $0.01 \%$ and the angle error is about $0.005 \%$, with high calibration accuracy. The actual measurement results show that this method can effectively calibrate the sound source azimuth, the error rate of the source coordinates is around $10 \%$, the horizontal declination angle error is less than $5 \%$, and the elevation angle error is less than $8 \%$. Appropriately increasing the spacing of the array will have a better calibration effect in an actual complex experimental environment.

\section{Introduction}

Sound is everywhere, along with people's daily life. In recent years, with industrial, civil, and military field, a sharp increase in demand for the positioning system, voice recognition systems [1,2], and sound source detection system [3] has become a new research hotspot. A sound localization system is actually the first to receive through a device the sound source signal, then the received signal, and sound source signal processing technology is adopted to improve the relevant technology to identify the target source location $[4,5]$. At present, the sound source positioning technology is mainly divided into two types [6]: active sound source positioning and passive sound source positioning. Compared with active sound source positioning technology, passive sound source positioning technology has strong concealment and anti- interference. This paper is based on a passive sound source positioning algorithm. It is difficult to locate the sound source rapidly and precisely because of the current sound source localization method, which directly affects the azimuth calibration of the sound source. In order to solve the above problems, we should improve the sound source localization accuracy in a complex environment and introduce a sound source azimuth calibration method, which has great practical significance.

Traditional sound source localization technology is mainly used in speech signal processing and military field $[7,8]$. Due to the foreign investment in the technology, the starting point is earlier than our country, so the foreign application in this technology is relatively mature. Equipment such as the UK Ferranti company Picker helicopter ISC company equipment alarm system, the United States' PALS 
ERAM remote passive acoustic positioning system (GPS), and the United States anti-vehicle intelligent mines [9], and so on, all the success of different applications in the field of military equipped with our R\&D and design of passive acoustic positioning system, has the higher performance of the system and has more ability of target detection and recognition. In the field of speech signal processing, foreign researchers have conducted a lot of in-depth research. Oppenheim and Lim [10] and Oppenheim et al. [11, 12] also demonstrated the contribution of phase-only signal reconstruction on human perception with regard to the quality of the reconstructed signal, exemplified for audio, image, crystallographic, and speech processing applications. Liu et al. studied the perceptual importance of the phase spectrum specifically on the intervocalic stop consonants, and they also reported the strong dependency of the perception of intervocalic stops on phase information [13]. In 1985, Flanagan, in a large conference speech enhancement in the application of the speech signal processing technology, obtained the good actual effect. In 1992, Silverman applied the technology to the speaker's speech recognition system, and it also achieved good results. In addition, the research on the acoustic source localization algorithm also has a great breakthrough, in which the beamforming method based on the microphone array $[14,15]$ appears early and can be applied in practice. In view of the limited resolution of the problems of the algorithm, after in-depth analysis and research, scholars put forward the algorithm of high resolution [16, 17], such as the minimum variance method, the characteristics of the structure method, and the signal subspace method [18].

Further research by researchers Paliwal and Alsteris [19] elaborates and demonstrates the importance of phase information in human hearing. Alsteris and Paliwal [20] show that the short-term phase spectrum can significantly improve the speech articulation in a small window of 20 to 40 milliseconds, which is very useful. Alsteris and Paliwal [21] based on automatic speech recognition (ASR) proved that in all cases, the resolution is superior to short weight of stimulus provided by the clarity, short time of amplitude, and phase spectrum information knowledge which lead to superior human speech recognition performance. Paliwal et al. [22] reported based on the analysis of the match or not match window for AMS during the processing of the amplitude and phase spectrum estimation and phase spectrum compensation (PSC) method that the minimum mean square error (MMSE) short-term spectrum amplitude (STSA) estimation to replace the noise amplitude spectrum and improve the voice quality is estimated based on the accurate phase spectrum. The overview of microphone array speech processing is proposed by Brandstein and Ward [23] in 2001, and it is considered that the phase difference between channels is the main source of information for delay estimation and source location application. Kudriashov [24] proposed the basic method for the three-dimensional positioning of sound source in distance-span-elevation coordinates by combining the time delay estimation results.

Although the research in the field of sound source localization technology in our country lags behind that of advanced countries but with the rapid development of modern technology in our country, the researchers are also actively involved in the study of the technology. Under the support of the National Happiness Technology 863, some institutions in our country have carried out the research on the smart bomb [25]. There is no lack of some technical strength of the high-tech enterprises and study of the sound source localization technology, represented by the company, establishing a sound source localization based on microphone array product research and development projects. Ju et al. [26] combine the characteristics of the subarray algorithm and the microphone array signal processing in the far-field narrowband signal and propose the sound source $3 \mathrm{D}$ localization algorithm based on the microphone array. Ju et al. [27] extended the classical MUSIC algorithm to the speech array processing to realize the three-dimensional localization of the sound source. Ding et al. [28] studied the discharge characteristic of the air gap to the level of the high altitude air gap discharge voltage calibration which provides basis, but with the increase of altitude, rod-plate and barbar clearance operation impact discharge voltage is reduced, and bar-bar discharge gap voltage margin lower than the error bar-plate gap is enlarged. In Xing et al.'s paper [29], in the data of the atmospheric electric field calibration method, in order to improve the consistency of the electric network data, the scope of the thunderstorm monitoring is expanded, to effectively solve the problem of the observatory data unification and avoid a severe distortion in electric network; the calibration method is limited to an altitude of the vertical direction but is not from the perspectives of atmospheric electric field instrument observation of thunder cloud monitoring. Wang et al. [30] put forward a kind of array by using auxiliary array element each dependent on an amplitude-phase error since the new method of correction is applicable to arbitrary array geometry, and its computational complexity is small, but only limited to the two-dimensional space.

Based on the acoustic source geometry localization algorithm, this paper studies the sound source omnidirectional positioning calibration method based on microphone observation angle. Based on the array, the relative time delay of a pair of microphones on the vertical axis is used to realize the omnidirectional sound source location. The array parameters are analyzed, and the improved method is put forward, using the data from the angle which measured sound source array based on observation angle of view, on the basis of the forward modeling data, carried out omnidirectional positioning calibration of the sound source, and analyzed the simulation data and actual measurement experiment in a complex real environment.

\section{Seven-Element Microphone Array Model}

The sound source geometric localization algorithm [31-33] and position calibration methods $[29,30,34]$ are based on a microphone array model [23,35-38]; the establishment of a reasonable set microphone array model and array element spacing can better match algorithm, reduce the complexity 


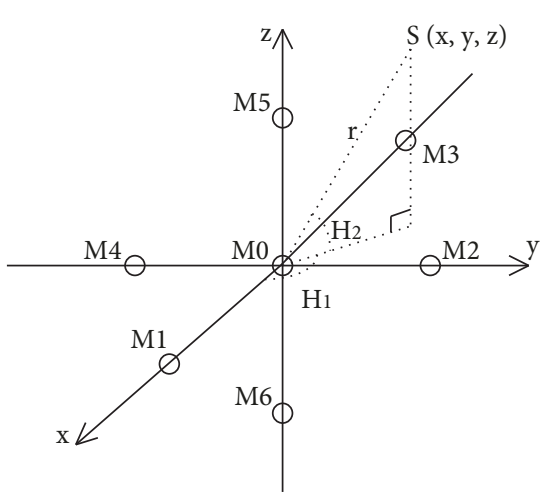

Figure 1: Sound source $S$ is not on the $\mathrm{x}-0-\mathrm{z}$ plane, $0-\mathrm{y}-\mathrm{z}$ plane, and $z$-axis.

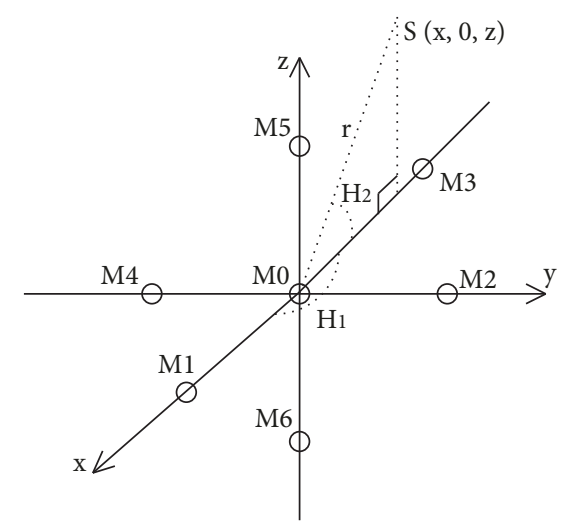

FIgURE 2: Sound source $S$ on the $\mathrm{x}-0-\mathrm{z}$ plane.

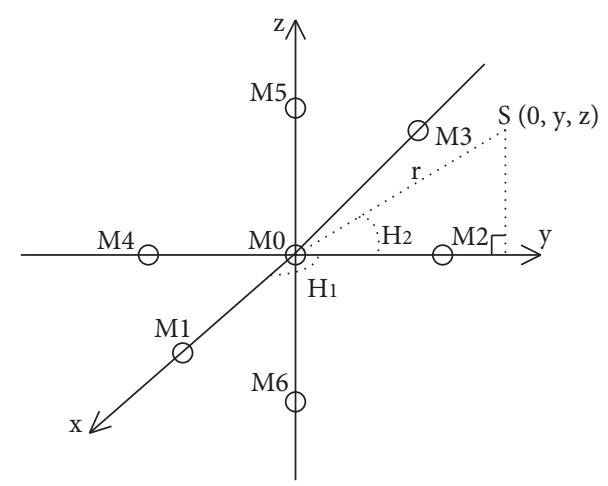

FIgURE 3: Sound source $S$ on the $0-y-z$ plane.

of the calculation, and improve the efficiency of the operation and sound comprehensive orientation calibration precision.

A seven-element microphone array model was established, and seven microphones were used to compose the array as shown in Figures 1-4: Figure 1 shows the array model where the sound source $S$ is not on the $\mathrm{x}-0-\mathrm{z}$ plane, 0 -y-z plane, and $z$-axis; Figure 2 shows the array model of the sound source $S$ on the $\mathrm{x}-\mathrm{O}-\mathrm{z}$ plane; Figure 3 shows the array model of the sound source $S$ on the $0-y-z$ plane; Figure 4 shows the array model of the sound source $S$ on

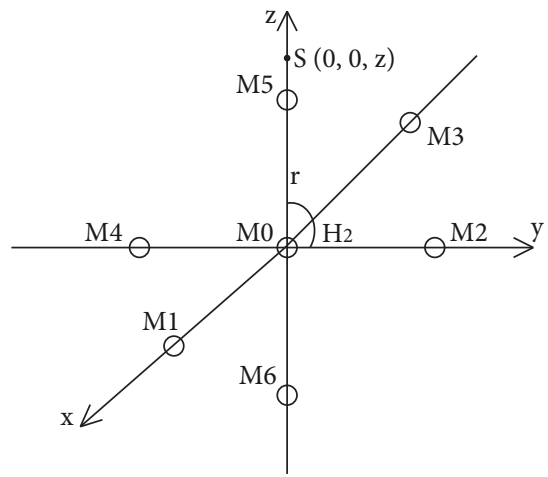

FIgURE 4: Sound source $S$ on the $z$-axis.

the $z$-axis. The sound source propagation velocity is $c$, and the space position of sound source $S$ is $(x, y, z)$, and $r$ is the distance from the sound source to the array center M0. The time for $S$ to propagate to microphones $\mathrm{M} 0(0,0,0)$, M1(a,0,0), M2(0,a,0), M3(-a,0,0), M4(0,-a,0), M5(0,0,a), and $M 6(0,0,-a)$ is $t 0, t 1, t 2, t 3, t 4, t 5$, and $t 6$, respectively. Based on the model, the relative delay values of 5 groups are set: $T_{1}=\mathrm{t} 1-\mathrm{t} 0, T_{2}=\mathrm{t} 2-\mathrm{t} 0, T_{3}=\mathrm{t} 3-\mathrm{t} 0, T_{4}=\mathrm{t} 4-\mathrm{t} 0$, and $T_{5}=\mathrm{t} 6-\mathrm{t} 5 . H_{1}$ is the horizontal deflection of the sound source $S$ relative to the $x$-positive half axis, and the range is between 0 and 360 degrees. $H_{2}$ is the elevation angle formed by the sound source $S$ and the $\mathrm{x}-\mathrm{y}-0$ plane (range between -90 and 90 degrees). If the value of $\mathrm{H}_{2}$ is positive, that is, $T_{5}$ is greater than zero, it means that the sound source is above the $\mathrm{x}-\mathrm{y}-0$ plane. If it is negative, that is, $T_{5}$ is less than zero, it means that the sound source is below the $x-y-0$ plane; this is the key to realizing the omnidirectional sound source positioning calibration.

\section{Sound Source Geometric Localization Algorithm}

A suitable microphone array model can be established in order to obtain a smaller error and further prepare the omnidirectional sound source positioning calibration. The localization algorithm of sound source azimuth needed to undertake choosing according to the array structure and adopt different localization algorithms will have different positioning precisions and computing speeds, accompanied by the algorithm of the computation size and position precision of algorithm.

Based on the location method of arrival delay, there is a maximum likelihood method based on search [39-41] and geometric localization algorithm [31-33]: the search method has a large amount of calculations and does not apply to real-time systems; if the sound source geometric localization algorithm is adopted, the algorithm has high positioning accuracy and the arrival delay itself has strong anti-interference, which can reduce the influence of the attenuation and interference of the actual sound propagation process on the accuracy of the sound source omnidirectional positioning calibration. 
3.1. Sound Source S Is Not on the $x-0-z$ Plane, $0-y-z$ Plane, and $z$-Axis. According to Figure 1, the seven-element microphone array model whose sound source is not on the $\mathrm{x}-0-\mathrm{z}$ plane, $0-\mathrm{y}-$ $\mathrm{z}$ plane, and $z$-axis represents the distance from the sound source $S$ to M0, M1, M2, M3, and M4, and its expression is as follows:

$$
\left\{\begin{array}{l}
x^{2}+y^{2}+z^{2}=r^{2}, \\
(x-a)^{2}+y^{2}+z^{2}=\left(r+c T_{1}\right)^{2}, \\
x^{2}+(y-a)^{2}+z^{2}=\left(r+c T_{2}\right)^{2}, \\
(x+a)^{2}+y^{2}+z^{2}=\left(r+c T_{3}\right)^{2}, \\
x^{2}+(y+a)^{2}+z^{2}=\left(r+c T_{4}\right)^{2} .
\end{array}\right.
$$

$x, y$, and $z$ are the sound source coordinate parameters of the sound source geometric localization algorithm based on the array of seven-element microphones, and formula (1) is the linear analysis basis of the omnidirectional positioning calibration method of sound source. According to (1), it can be concluded that

$$
\left\{\begin{array}{l}
x^{\prime}=\frac{f\left(4 a^{2}-c^{2} n+c^{2} m e\right)}{4 a m}, \\
y^{\prime}=\frac{g\left[4 a^{2}-c^{2} n+c^{2} m(m-e)\right]}{4 a m} .
\end{array}\right.
$$

Among them, $m=T_{1}+T_{2}+T_{3}+T_{4}, \quad n=T_{1}{ }^{2}+T_{2}{ }^{2}+$ $T_{3}{ }^{2}+T_{4}{ }^{2}, e=T_{1}+T_{3}, f=T_{3}-T_{1}, g=T_{4}-T_{2}$, parameter $x^{\prime}$ is the simulation value of the sound source coordinate parameter $x$, and parameter $y^{\prime}$ is the simulation value of the source coordinate parameter $y$.

According to the actual situation, since the magnitudes of sum $e=T_{1}+T_{3}$ and $m-e=T_{2}+T_{4}$ and sum $m$ are both about $10^{-6}$, then the order of magnitude $c^{2} m e$ is $10^{-7}$, and the order of magnitude $4 a^{2}-c^{2} n$ is only $10^{1}$; relatively speaking, $c^{2} m e$ can be ignored, and the same reason $c^{2} m(m-e)$ can be ignored, so (2) can be simplified for

$$
\left\{\begin{array}{l}
x^{\prime}=\frac{\left(T_{3}-T_{1}\right)\left(4 a^{2}-c^{2} n\right)}{4 a m}, \\
y^{\prime}=\frac{\left(T_{4}-T_{2}\right)\left(4 a^{2}-c^{2} n\right)}{4 a m} .
\end{array}\right.
$$

Using (1) again, we can get the expression of the distance from the sound source to the center of the array M0, that is,

$$
r^{\prime}=\frac{4 a^{2}-c^{2} n}{2 m c}
$$

In the formula, $a$ is the distance between the array elements, $c$ is the sound source propagation speed, and $r^{\prime}$ is the simulation parameter of the sound source coordinate parameter $r$. $a$ and $c$ are theoretical constants. Since (3) and (4) are only related to relative delay value $T_{1}$ to $T_{4}$, they can be used in simulation experiments for the sound source omnidirectional positioning calibration method and error analysis for the localization accuracy of sound source.

According to Figure 1, the seven-element microphone array model where the sound source $S$ is not on the $\mathrm{x}-0-\mathrm{z}$ plane, $0-y-z$ plane, and $z$-axis can be used to derive the correlation equation:

$$
\left\{\begin{array}{l}
x^{\prime}=r^{\prime} \cos H_{2}{ }^{\prime} \cos H_{1}{ }^{\prime}, \\
y^{\prime}=r^{\prime} \cos H_{2}{ }^{\prime} \sin H_{1}{ }^{\prime}, \\
z^{\prime}=r^{\prime} \sin H_{2}{ }^{\prime} .
\end{array}\right.
$$

Equation (5) based on the polar coordinate relationship of the three-dimensional geometry is the theoretical basis for solving the horizontal declination angle and the elevation angle. Among them, $H_{1}{ }^{\prime}$ is the simulation value of the horizontal declination angle $H_{1}$ in the omnidirectional positioning of the sound source, $\mathrm{H}_{2}{ }^{\prime}$ is the simulation value of the elevation angle $\mathrm{H}_{2}, r^{\prime}$ is the simulation value of the acoustic source coordinate parameter $r$, and $z^{\prime}$ is the simulation value of the sound source coordinate parameter $z$.

Using (5), the value of horizontal declination $H_{1}{ }^{\prime}$ is

$$
\left\{\begin{array}{l}
H_{1}^{\prime}=\arctan \left(\frac{y^{\prime}}{x^{\prime}}\right), \quad x^{\prime}>0, \\
H_{1}{ }^{\prime}=180+\arctan \left(\frac{y^{\prime}}{x^{\prime}}\right), \quad x^{\prime}<0 .
\end{array}\right.
$$

According to the positive and negative of $T_{5}$, the elevation value $\mathrm{H}_{2}{ }^{\prime}$ can be obtained by polarity judgment. Using (5) again, the following expression can be obtained:

$$
\begin{cases}H_{2}{ }^{\prime}=\arccos \frac{x^{\prime}}{r^{\prime} \cos H_{1}{ }^{\prime}}, & T_{5}>0, \\ H_{2}{ }^{\prime}=-\arccos \frac{x^{\prime}}{r^{\prime} \cos H_{1}{ }^{\prime}}, & T_{5}<0 .\end{cases}
$$

In the formula, $T_{5}$ is the time delay between the sound source $S$ arriving at the microphones M5 and M6. After getting $H_{2}{ }^{\prime}$ according to the polarity judgment of $T_{5}$, (5) is used to determine the sound source coordinate parameter $z^{\prime}$, and then the parameters $x^{\prime}$ and $y^{\prime}$ are combined to realize the omnidirectional positioning of the sound source and further be used for omnidirectional positioning calibration of the sound source.

Based on the seven-element microphone array model shown in Figure 1, the theoretical angle value can be solved, and its expression is as follows: 


$$
\left\{\begin{array}{l}
H_{1}=\arctan \frac{y}{x}, \quad x>0, \\
H_{1}=180+\arctan \frac{y}{x}, \quad x<0, \\
H_{1}=90^{\circ}, \quad x=0, y>0, \\
H_{1}=-90^{\circ}, \quad x=0, y<0, \\
H_{2}=\arcsin \frac{z}{r} .
\end{array}\right.
$$

3.2. Sound Source $S$ Is on the $x-0-z$ Plane. According to the seven-element microphone array model of the sound source $S$ on the $\mathrm{x}-0-\mathrm{z}$ plane in Figure 2, because only the value of the sound source coordinate parameter $y^{\prime}$ is changed, when $y^{\prime}$ is equal to 0 and $x^{\prime}$ and $z^{\prime}$ are not 0 , which is equivalent to only changing the time delay value $T_{1}$ to $T_{5}$, using (3) and (4), the expression of the sound source coordinate parameters $x^{\prime}, y^{\prime}$, and $z^{\prime}$ is as follows:

$$
\left\{\begin{array}{l}
x^{\prime}=\frac{\left(T_{3}-T_{1}\right)\left(4 a^{2}-c^{2} n\right)}{4 a m} \\
y^{\prime}=0 \\
r^{\prime}=\frac{4 a^{2}-c^{2} n}{2 m c}
\end{array}\right.
$$

Based on Figure 2, the value of horizontal declination $H_{1}{ }^{\prime}$ can be obtained, and its expression is as follows:

$$
\left\{\begin{array}{l}
H_{1}{ }^{\prime}=0^{\circ}, \quad x^{\prime}>0, \\
H_{1}{ }^{\prime}=180^{\circ}, \quad x^{\prime}<0 .
\end{array}\right.
$$

From (10), it can be seen that the value of $H_{1}{ }^{\prime}$ is completely determined by the positive and negative values of the sound source coordinate parameter $x^{\prime}$, which conforms to the solid geometry shown in Figure 2.

According to the time delay value $T_{5}$ and the positive and negative of the sound source coordinate parameter $x^{\prime}$, the elevation value $\mathrm{H}_{2}{ }^{\prime}$ can be judged by the polarity and the value $\mathrm{H}_{2}{ }^{\prime}$ can be obtained by using formula (5). Its expression is as follows:

$$
\left\{\begin{array}{l}
H_{2}^{\prime}=\arccos \frac{x^{\prime}}{r^{\prime}}, \quad T_{5}>0, x^{\prime}>0, \\
H_{2}^{\prime}=180-\arccos \frac{x^{\prime}}{r^{\prime}}, \quad T_{5}>0, x^{\prime}<0, \\
H_{2}^{\prime}=-\arccos \frac{x^{\prime}}{r^{\prime}}, \quad T_{5}<0, x^{\prime}>0, \\
H_{2}^{\prime}=\arccos \frac{x^{\prime}}{r^{\prime}}-180, \quad T_{5}<0, x^{\prime}<0 .
\end{array}\right.
$$

Similarly, after determining $H_{2}{ }^{\prime}$ according to the polarities of $T_{5}$ and $x^{\prime}$, use (5) to determine the sound source coordinate parameter $z^{\prime}$ and combine parameters $x^{\prime}$ and $y^{\prime}$ to achieve omnidirectional localization of the sound source and further use it for the sound source omnidirectional positioning calibration. Using (8), the theoretical values of horizontal declination $H_{1}$ and elevation $H_{2}$ can be solved.

3.3. Sound Source S Is on the 0-y-z Plane. According to the seven-element microphone array model of the sound source $S$ on the $0-y-z$ plane in Figure 3, because only the value of the sound source coordinate parameter $x^{\prime}$ is changed, when $x^{\prime}$ is equal to 0 and $y^{\prime}$ and $z^{\prime}$ are not 0 , which is equivalent to only changing the time delay value $T_{1}$ to $T_{5}$, again using (3) and (4), the expression of the sound source coordinate parameters $x^{\prime}, y^{\prime}$, and $z^{\prime}$ is as follows:

$$
\left\{\begin{array}{l}
x^{\prime}=0 \\
y^{\prime}=\frac{\left(T_{4}-T_{2}\right)\left(4 a^{2}-c^{2} n\right)}{4 a m} \\
r^{\prime}=\frac{4 a^{2}-c^{2} n}{2 m c}
\end{array}\right.
$$

Based on Figure 3, the value of horizontal declination $H_{1}{ }^{\prime}$ can be obtained, and its expression is as follows:

$$
\begin{cases}H_{1}{ }^{\prime}=90^{\circ}, & y^{\prime}>0, \\ H_{1}{ }^{\prime}=-90^{\circ}, & y^{\prime}<0 .\end{cases}
$$

From (13), it can be seen that the value of $H_{1}{ }^{\prime}$ is completely determined by the positive and negative values of the sound source coordinate parameter $y^{\prime}$, which conforms to the solid geometry shown in Figure 3.

According to the time delay value $T_{5}$ and the positive and negative of the sound source coordinate parameter $y^{\prime}$, the elevation value $\mathrm{H}_{2}{ }^{\prime}$ can be judged by the polarity and the value $\mathrm{H}_{2}{ }^{\prime}$ can be obtained by using formula (5). Its expression is as follows:

$$
\left\{\begin{array}{l}
H_{2}{ }^{\prime}=\arccos \frac{y^{\prime}}{r^{\prime}}, \quad T_{5}>0, y^{\prime}>0, \\
H_{2}{ }^{\prime}=180-\arccos \frac{y^{\prime}}{r^{\prime}}, \quad T_{5}>0, y^{\prime}<0, \\
H_{2}{ }^{\prime}=-\arccos \frac{y^{\prime}}{r^{\prime}}, \quad T_{5}<0, y^{\prime}>0, \\
H_{2}{ }^{\prime}=\arccos \frac{y^{\prime}}{r^{\prime}}-180, \quad T_{5}<0, y^{\prime}<0, \\
z^{\prime}=r^{\prime} \sin H_{2}{ }^{\prime} .
\end{array}\right.
$$

Similarly, after determining $\mathrm{H}_{2}{ }^{\prime}$ according to the polarities of $T_{5}$ and $y^{\prime}$, use (5) to determine the sound source coordinate parameter $z^{\prime}$ and combine parameters $x^{\prime}$ and $y^{\prime}$ to achieve omnidirectional localization of the sound source and further use it for the sound source omnidirectional 


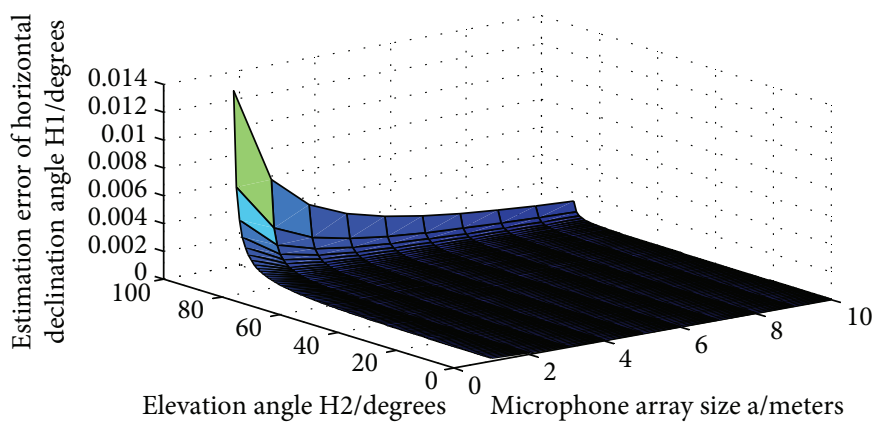

FIgURE 5: Estimation error of the horizontal declination angle $H_{1}$ vs. array size $a$ and elevation angle $H_{2}$.

positioning calibration. Again using (8), the theoretical values of horizontal declination $H_{1}$ and elevation $H_{2}$ can be solved.

3.4. Sound Source S Is on the z-Axis. According to the sevenelement microphone array model of the sound source $S$ on the $z$-axis in Figure 4, because only the values of the sound source coordinate parameters $x^{\prime}$ and $y^{\prime}$ are changed and $x^{\prime}$ and $y^{\prime}$ are both 0 and $z^{\prime}$ are not 0 at this time, which is equivalent to changing only the time delay value $T_{1}$ to $T_{5}$. By using (3) and (4), the expressions for parameters $x^{\prime}, y^{\prime}$, and $z^{\prime}$ are as follows:

$$
\left\{\begin{array}{l}
x^{\prime}=0, \\
y^{\prime}=0 \\
r^{\prime}=\frac{4 a^{2}-c^{2} n}{2 m c} .
\end{array}\right.
$$

Based on Figure 4, the value of horizontal declination $H_{1}{ }^{\prime}$ can be obtained, and its expression is as follows:

$$
H_{1}{ }^{\prime}=0^{\circ}
$$

According to the positive and negative values of the time delay value $T_{5}$, the values of the elevation angle value $\mathrm{H}_{2}{ }^{\prime}$ and the sound source coordinate parameter $z^{\prime}$ can be determined and obtained, and the related expressions are as follows:

$$
\left\{\begin{array}{l}
z^{\prime}=r^{\prime}, H_{2}{ }^{\prime}=90^{\circ}, \quad T_{5}>0, \\
z^{\prime}=-r^{\prime}, H_{2}{ }^{\prime}=-90^{\circ}, \quad T_{5}<0 .
\end{array}\right.
$$

Again using (8), the theoretical values of horizontal declination $H_{1}$ and elevation $H_{2}$ can be solved.

\section{Error Analysis of Sound Source Positioning Calibration and the Way to Reduce the Error}

Through the analysis of azimuth positioning formulas (3) and (4), it can be known that the azimuth positioning performance of the target sound source is related to the time delay estimation error, the size of the microphone array, and the effective speed of sound.

The statistical characteristics of time delay are the same, assuming that the standard deviation of the time delay estimation error is $\xi_{T}$ and independent of each other.

4.1. Horizontal Declination Angle Error Analysis. The estimation error of the horizontal declination angle $H_{1}$ caused by the delay estimation error is

$\xi_{H_{1} T}=\sqrt{\left(\frac{\partial H_{1}}{\partial T_{1}}\right)^{2} \xi_{T}^{2}+\left(\frac{\partial H_{1}}{\partial T_{2}}\right)^{2} \xi_{T}^{2}+\left(\frac{\partial H_{1}}{\partial T_{3}}\right)^{2} \xi_{T}^{2}+\left(\frac{\partial H_{1}}{\partial T_{4}}\right)^{2} \xi_{T}^{2}}$.

Using $H_{1}=\arctan \left(\left(T_{4}-T_{2}\right) /\left(T_{3}-T_{1}\right)\right)$, one can obtain the partial derivative of the horizontal declination angle $H_{1}$ relative to the delay value $T_{1}$ to $T_{4}$, and its expression is as follows:

$$
\left\{\begin{array}{l}
\frac{\partial H_{1}}{\partial T_{1}}=-\frac{\partial H_{1}}{\partial T_{3}}=\frac{1}{1+\tan ^{2} H_{1}} \cdot \frac{T_{4}-T_{2}}{\left(T_{3}-T_{1}\right)^{2}}, \\
\frac{\partial H_{1}}{\partial T_{2}}=-\frac{\partial H_{1}}{\partial T_{4}}=-\frac{1}{1+\tan ^{2} H_{1}} \cdot \frac{1}{T_{3}-T_{1}}
\end{array}\right.
$$

Equation (19) can be used to derive the equation for the estimation error $\xi_{H_{1} T}$ of horizontal declination angle $H_{1}$ caused by the delay estimation error, which is

$$
\xi_{H_{1} T}=\frac{\sqrt{2} c \xi_{T}}{2 a \cos H_{2}}
$$

Equation (20) is simulated. When the estimation error at the time delay is $1 \mu \mathrm{s}$, the error curve shown in Figure 5 can be obtained.

As can be seen from Figure 5, the accuracy of the horizontal declination angle estimation has nothing to do with the horizontal declination angle value, but it is affected by the time delay estimation error, the microphone array size, and the elevation angle value. When the size of the microphone array $a$ increases, the estimation error of the horizontal declination angle $H_{1}$ decreases; between the estimation errors of the horizontal declination angle $H_{1}$ and the elevation angle $\mathrm{H}_{2}$, the estimation error of the horizontal 


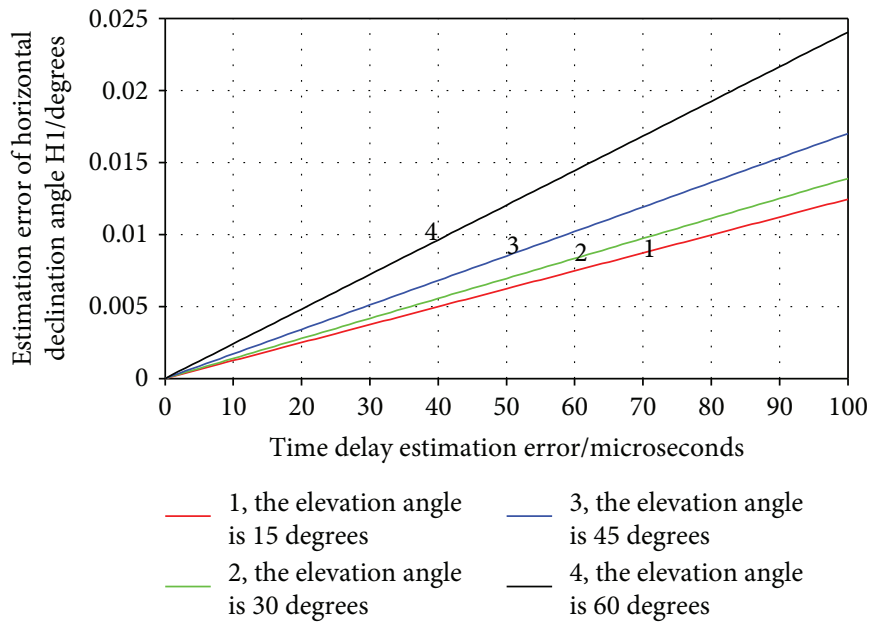

FIgURE 6: The relationship between the estimation error of horizontal declination angle $H_{1}$ and the time delay estimation error.

declination angle decreases as the elevation angle decreases. Therefore, this is a more accurate measurement of lowaltitude and ground sound sources than aerial sound source measurements.

When the array spacing $a$ is equal to 2 meters and the elevation angles are 15 degrees, 30 degrees, 45 degrees, and 60 degrees, the relationship between the estimation error of horizontal declination angle $H_{1}$ and the time delay estimation error is shown in Figure 6.

From Figure 6, we can see that when the value of the elevation $\mathrm{H}_{2}$ is certain, the estimation error of horizontal declination angle $H_{1}$ increases linearly with the increase of the time delay estimation error; as the elevation angle value $\mathrm{H}_{2}$ increases, the linear variation between the two increases more sharply, indicating that the estimation error of the delay has a greater influence on the measurement accuracy of the horizontal declination angle $H_{1}$, and its measurement accuracy may drastically decrease.

4.2. Elevation Error Analysis. The estimation error of the elevation angle $\mathrm{H}_{2}$ caused by the delay estimation error is

$\xi_{H_{2} T}=\sqrt{\left(\frac{\partial H_{2}}{\partial T_{1}}\right)^{2} \xi_{T}^{2}+\left(\frac{\partial H_{2}}{\partial T_{2}}\right)^{2} \xi_{T}^{2}+\left(\frac{\partial H_{2}}{\partial T_{3}}\right)^{2} \xi_{T}^{2}+\left(\frac{\partial H_{2}}{\partial T_{4}}\right)^{2} \xi_{T}^{2}}$.

Using $H_{2}=\arccos \left((c / 2 a) \cdot \sqrt{\left(T_{3}-T_{1}\right)^{2}+\left(T_{4}-T_{2}\right)^{2}}\right)$, one can find the partial derivative of the elevation angle $H_{2}$ with respect to the delay value $T_{1}$ to $T_{4}$, which has the following expression:

$$
\left\{\begin{array}{l}
\frac{\partial H_{2}}{\partial T_{1}}=-\frac{\partial H_{2}}{\partial T_{3}}=\frac{c^{2}\left(T_{3}-T_{1}\right)}{2 a^{2} \sin \left(2 \cdot H_{2}\right)}, \\
\frac{\partial H_{2}}{\partial T_{2}}=-\frac{\partial H_{2}}{\partial T_{4}}=\frac{c^{2}\left(T_{4}-T_{2}\right)}{2 a^{2} \sin \left(2 \cdot H_{2}\right)}
\end{array}\right.
$$

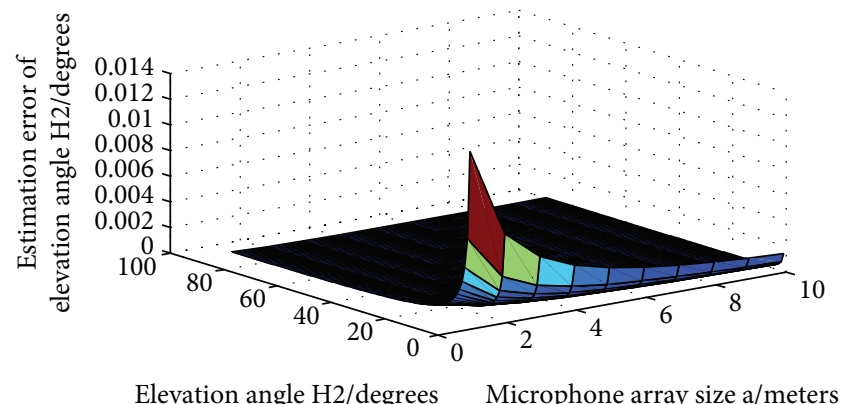

Figure 7: Estimation error of the elevation angle $\mathrm{H}_{2}$ vs. array size $a$ and elevation angle $\mathrm{H}_{2}$.

Equation (22) can be used to derive the equation for the estimation error $\xi_{\mathrm{H}_{2} \mathrm{~T}}$ of the elevation angle $\mathrm{H}_{2}$ caused by the delay estimation error, which is

$$
\xi_{H_{2} T}=\frac{\sqrt{2} c \xi_{T}}{2 a \sin H_{2}} .
$$

Equation (23) is simulated. When the estimation error at the time delay is $1 \mu \mathrm{s}$, the error curve shown in Figure $7 \mathrm{can}$ be obtained:

As can be seen from Figure 7, the estimation accuracy of the elevation angle has nothing to do with the horizontal declination value, but it is also affected by the time delay estimation error, the microphone array size, and the elevation angle value. When the size of the microphone array $a$ increases, the estimation error of the elevation angle $\mathrm{H}_{2}$ decreases; the elevation angle $\mathrm{H}_{2}$ itself changes the angle of elevation, and as the elevation angle increases, the estimation error of the elevation angle decreases, that is, the accuracy of elevation angle measurement will increase.

When the array spacing $a$ is equal to 2 meters and the elevation angles are 15 degrees, 30 degrees, 45 degrees, and 60 degrees, the relationship between the estimation error of elevation angle $\mathrm{H}_{2}$ and the time delay estimation error is shown in Figure 8. 


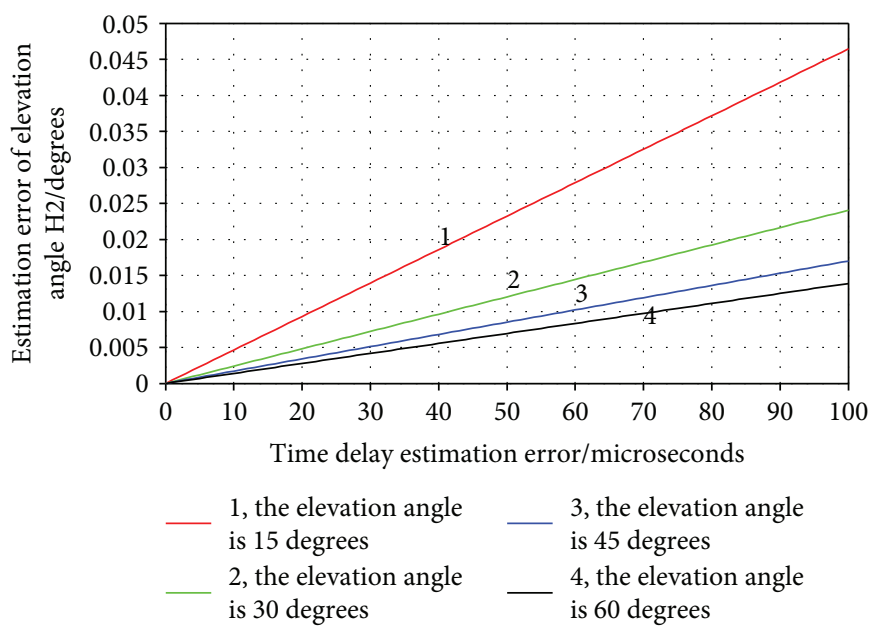

FIGURE 8: The relationship between the estimation error of elevation angle $\mathrm{H}_{2}$ and the time delay estimation error.

Figure 8 shows that when the value of elevation angle $\mathrm{H}_{2}$ is certain, its own estimation error will increase linearly with the increase of time delay estimation error; as the value of elevation angle $\mathrm{H}_{2}$ decreases, the linear change between the two will become more severe, indicating that the time delay estimation error has a greater influence on the accuracy of the elevation angle $\mathrm{H}_{2}$, and its measurement accuracy may drastically decrease.

4.3. Error Analysis of the Distance from the Sound Source to the Microphone Array Center. The standard deviation of the estimation error of the distance $r$ from the sound source to the center of the array is

$\xi_{r T}=\sqrt{\left(\frac{\partial r}{\partial T_{1}}\right)^{2} \xi_{T}^{2}+\left(\frac{\partial r}{\partial T_{2}}\right)^{2} \xi_{T}^{2}+\left(\frac{\partial r}{\partial T_{3}}\right)^{2} \xi_{T}^{2}+\left(\frac{\partial r}{\partial T_{4}}\right)^{2} \xi_{T}^{2}}$.

Once again using (1), the expression of any relative delay can be obtained as follows:

$$
T_{i}=\frac{1}{c}\left(\sqrt{r^{2}+a^{2}-2 r a \cos H_{2} \cos \left[H_{1}-\frac{\pi}{2}(i-1)\right]}-r\right)
$$

Among them, $i$ is equal to 1 to 4 , and $T_{i}$ is the relative delay of the sound source $S$ to reach the two microphones. From (4), it can be seen that the expression of the distance $r$ from the sound source to the array center M0 is related to $T_{i}$, and therefore, $T_{i}$ is solved first.

Let $(a / r)^{2}-(2 a / r) \cos H_{2} \cos \left[H_{1}-(\pi / 2)(i-1)\right]=x, a$ $\rightarrow 2 a$; equation (25) can be expressed as

$$
T_{i}=\frac{r}{c} \sqrt{1+4 x}-\frac{r}{c}
$$

Taylor's formula is used to expand (26) and ignore highorder infinitesimal parts, which is

$$
\begin{aligned}
T_{i} \approx & \frac{r}{c}\left(1+2 x-2 x^{2}\right)-\frac{r}{c} \\
= & \frac{r}{c}\left\{\left[2\left(\frac{a}{r}\right)^{2}-2 \frac{a}{r} \cos H_{2} \cos \left[H_{1}-\frac{\pi}{2}(i-1)\right]\right.\right. \\
& -2\left(\frac{a}{r}\right)^{2} \cos ^{2} H_{2} \cos ^{2}\left[H_{1}-\frac{\pi}{2}(i-1)\right\} .
\end{aligned}
$$

Let $\cos H_{2} \cos \left[H_{1}-(\pi / 2)(i-1)\right]=A$, where $i$ is equal to 1 to 4 and, at the same time, summed the two sides of the above equation. Because of $\sum_{i=1}^{4} A=0$ and $\sum_{i=1}^{4} A^{2}=\cos ^{2} H_{2}$, according to (27), the expression is as follows:

$$
\sum_{i=1}^{4} T_{i}=2 \frac{a^{2}}{r c}\left(4-\cos ^{2} H_{2}\right)
$$

According to (4) about the distance $r$ from the sound source to the center of the array M0, using (28), $r$ has the following expression:

$$
r=\frac{4 a^{2}-c^{2} n}{2 m c}=\frac{4 a^{2}-c^{2} \sum_{i=1}^{4} T_{i}^{2}}{2 c \sum_{i=1}^{4} T_{i}}
$$

Using (29) to find the relevant partial derivative and substituting it into (24), we can conclude that

$$
\xi_{r T}=\frac{r c}{a^{2}\left(4-\cos ^{2} H_{2}\right)} \sqrt{a^{2}+r^{2}} \xi_{T} .
$$

In (30), the relative ranging error $\xi_{r T}$ of the sound source is related to the accuracy of time delay estimation, the size of the microphone array, the distance $r$ from the sound source to the center of the array, and the elevation angle. However, irrelevant to the horizontal deviation angle, the variation of the horizontal deviation angle does not cause the change of relative ranging error. 


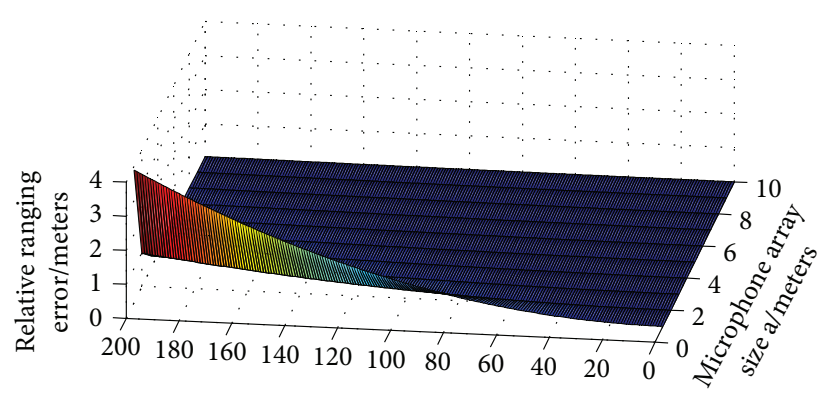

Distance from sound source to center of the array $\mathrm{r} / \mathrm{meters}$

FIGURE 9: The relation curve between the relative ranging error and the array size; the distance from the sound source to the center of the array.

Equation (30) is simulated, the elevation angle $H_{2}$ is 45 degrees, the delay estimation error is 1 microsecond, and the error curve shown in Figure 9 can be obtained.

As can be seen from Figure 9, when the time delay estimation accuracy and the microphone array size are certain, the relative ranging error increases as the distance $r$ from the sound source to the array center increases, and the relative ranging performance based on the array degrades. This situation is suitable for near-field sound source location; increasing the array size $a$, the relative range error is reduced, and the relative ranging accuracy will be improved, but the array size should not be less than 2 meters; otherwise, the relative ranging error will rise sharply.

When the elevation angle $\mathrm{H}_{2}$ is 30 degrees and 60 degrees, respectively, the relative ranging error is 0.05 radians and 0.1 radians, respectively, and the distance between the sound source and the center of the array is 100 meters; the relationship between the time delay estimation error and the microphone array size is shown in Figure 10.

As can be seen from Figure 10, when the relative ranging error, elevation angle $\mathrm{H}_{2}$, and microphone array size $a$ are combined and the microphone array size is constant, the relative ranging error has a greater influence on the time delay estimation error than the elevation angle size; when the size is large, the increase of the array size $a$ will cause an exponential increase in the error of the time delay estimation; especially when the size of the array is larger than 8 meters, the error increases more significantly. Therefore, it is recommended that the array size should not be too large.

When the elevation angles $\mathrm{H}_{2}$ are 30 degrees and 30 degrees, respectively, and the relative ranging error is 0.05 radians and 0.1 radians, respectively, and the array size is 2 meters, the relationship between the time delay estimation error and the distance from the sound source to the center of the array is shown in Figure 11.

It can be seen from Figure 11 that when the distance $r$ from the sound source to the center of the array is small, the relationship between it and the time delay estimation error changes drastically. In particular, when the sound source is very close to the center of the array, the time delay estimation error tends to be infinite; this agrees with the actual situation. This indicates that it is not appropriate to locate the sound source that is too close to the center of the array. Before integrating the error analysis, when the distance between the sound source and the array center reaches 8 to 10 meters, it will be better for the sound source to be fully calibrated.

4.4. Error Analysis of the Effect of Sound Velocity on Calibration Accuracy of Sound Source Location. The speed at which sonars travel in different media is different. At a temperature of $273.16 \mathrm{~K}$ and an atmospheric pressure of $105 \mathrm{~Pa}$, the speed of sound $c$ in the air is equal to 331.45 plus or minus 0.05 meters per second. In the actual environment, the influence of the ambient temperature on the speed of sound can not be ignored. Under the condition that the atmospheric pressure and other conditions remain relatively stable, the formula for calculating the sound velocity in the air at different temperatures is

$$
c=c_{0} \sqrt{\frac{T}{T_{0}}} .
$$

Among them, $c_{0}=331.45$ meters per second and $T_{0}=273.16 \mathrm{~K}$.

According to (31), relevant conclusions can be drawn: the speed of sound propagation in air is different at different temperatures, and the speed of sound increases with increasing temperature. Therefore, it is necessary to perform temperature measurement according to different environments during actual application to improve calibration accuracy.

4.5. The Way to Reduce the Error. Based on the error analysis of parameters such as the horizontal declination angle, elevation angle, the distance between the sound source and the center of the array, and sound velocity based on the sevenelement microphone array model, the following five-point error reduction method is proposed:

(1) From the analysis of the estimation error of the horizontal declination angle $H_{1}$ and the elevation angle $\mathrm{H}_{2}$, the microphone array size $a$ can be appropriately increased to reduce the estimation error of both, thereby improving the measurement accuracy of both

(2) From the analysis of the estimation error of the distance from the sound source to the center of the array, the size of the array can be appropriately increased to reduce the relative ranging error, thereby improving the relative ranging accuracy, and the general array size should not be less than 2 meters

(3) From the curve of the relation between array size $a$ and time delay estimation error, it can be seen that the general array size should not be larger than 10 meters

(4) It can be seen from the plot of the distance $r$ from the sound source to the center of the array element and the time delay estimation error that when $r$ reaches 8 to 10 meters, the sound source has a better omnidirectional positioning calibration effect 

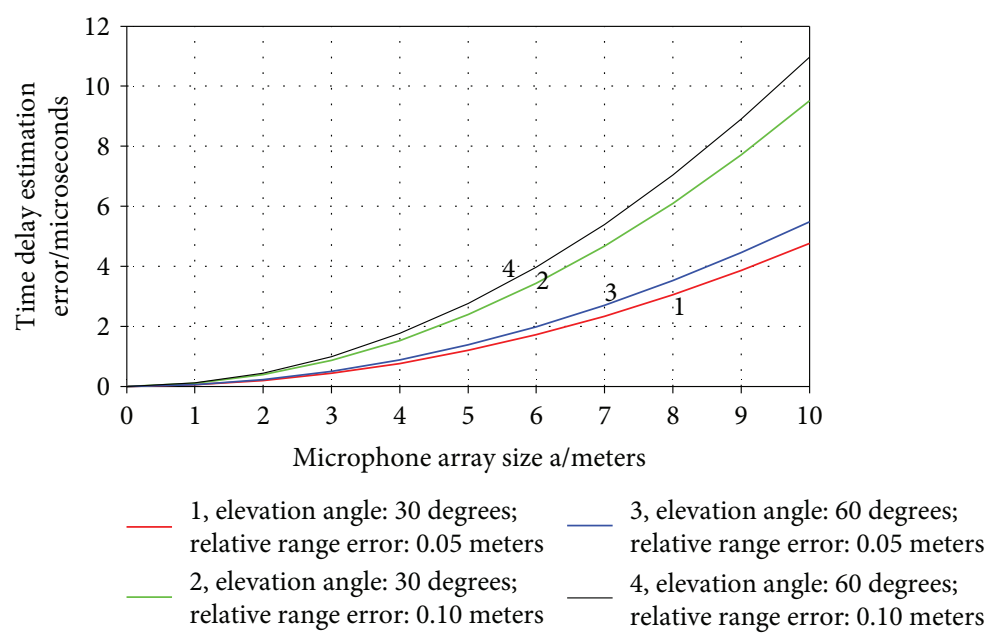

FIGURE 10: The relationship between microphone array size and time delay estimation error.
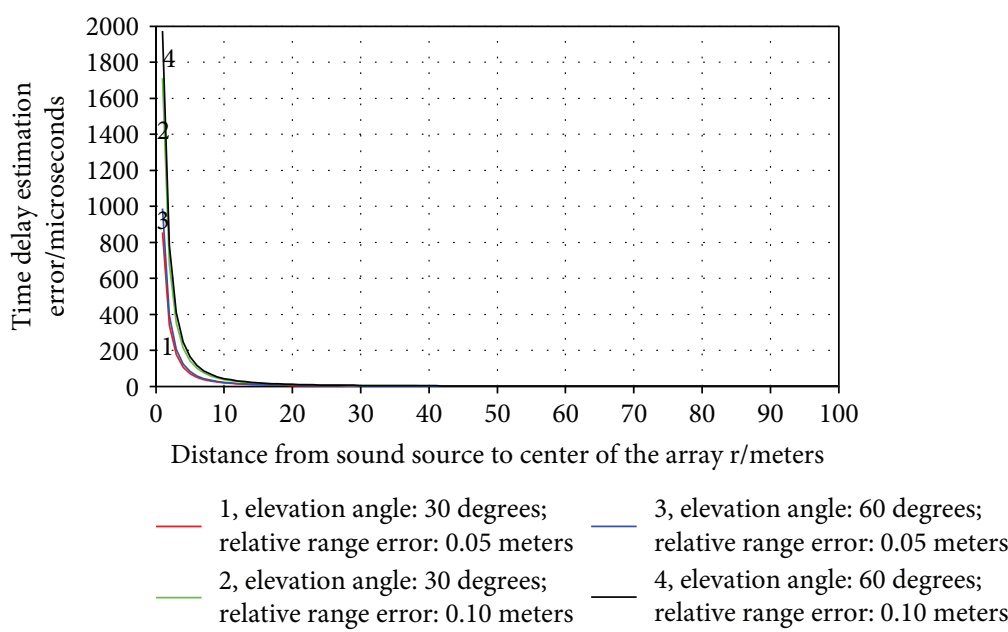

FIGURE 11: The relationship between distance from sound source to center of the array and time delay estimation error.

(5) Real-time measurement of actual temperature in different location environments and corresponding correction of sound speeds to reduce the error of sound source positioning calibration.

\section{Sound Source Omnidirectional Positioning Calibration}

Consider that in the actual environment, locating the sound source based on the microphone array cannot be directly transmitted to the spatial location information of the actual sound source of the observation party. Using the omnidirectional data of the sound source measured from the perspective of the array of seven-element microphones, a new coordinate system was rebuilt based on the observation angle based on the microphone, and the azimuth position of the sound source was calibrated.

5.1. Sound Source Positioning Calibration Model. Let the observation angle $N$ be located in the coordinate system of the original microphone array, and denote it as $(X, Y, Z)$.
The sound source positioning calibration model based on the observation angle of the microphone is shown in Figure 12.

According to Figure 12, we convert $(X, Y, Z)$ to $(0,0,0)$ and use this as a starting point to create a new coordinate system, converting $\left(x^{\prime}, y^{\prime}, z^{\prime}\right)$ to $\left(x^{\prime}-X, y^{\prime}-Y, z^{\prime}-Z\right)$, which can be equivalent to $\left(X^{\prime}, Y^{\prime}, Z^{\prime}\right)$. Let the angle between the sound source $S$ and the positive half of $x 1$ in the new coordinate system be the horizontal declination angle $h_{1}$ in the range of 0 to 360 degrees, and the angle between the source $S$ and the plane of the new coordinate system $(\mathrm{x} 1, \mathrm{y} 1,0)$ is the elevation angle $h_{2}$ (range from -90 to 90 degrees). If the value of $h_{2}$ is positive, it means that the sound source is above the plane of $N$; if it is negative, it is below it; the distance from $\left(X^{\prime}, Y^{\prime}, Z^{\prime}\right)$ to observation angle $N$ is $R^{\prime}$.

5.2. Sound Source Positioning Calibration Method. According to Figure 12, based on the sound source positioning calibration model, using the measured omnidirectional positioning coordinates of the sound source, no matter where the 


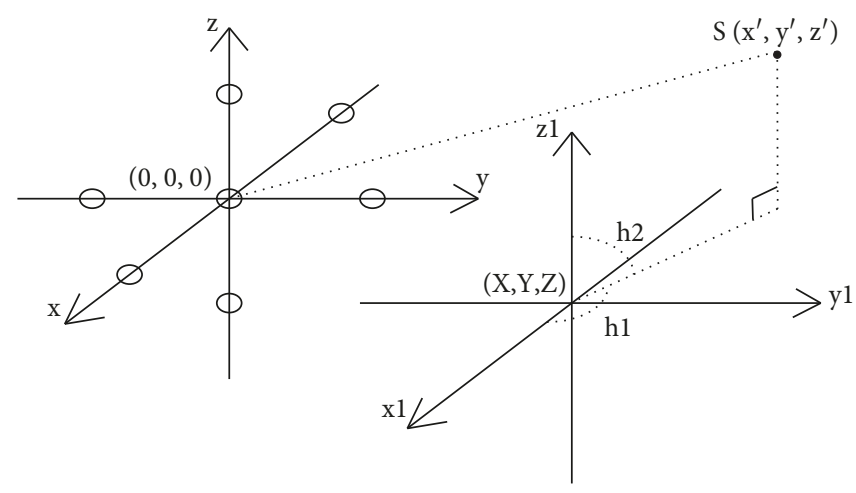

FIGURE 12: Sound source positioning calibration model based on microphone observation angle.

observation angle $N$ is located, the sound source positioning calibration can be performed. Its expression is as follows:

$$
\left\{\begin{array}{l}
X^{\prime}=x^{\prime}-X, \\
Y^{\prime}=y^{\prime}-Y, \\
Z^{\prime}=z^{\prime}-Z, \\
R^{\prime}=\sqrt{X^{\prime 2}+Y^{\prime 2}+Z^{\prime 2}} .
\end{array}\right.
$$

After using (32) to determine the omnidirectional positioning coordinates $\left(X^{\prime}, Y^{\prime}, Z^{\prime}\right)$ of the sound source based on the observation angle, according to the actual situation, using the analytical method of the three-dimensional geometry, determine the positive and negative coordinates of the sound source parameters $X^{\prime}$ and $Y^{\prime}$, and obtain the horizontal declination angle $h_{1}$ and the elevation angle $h_{2}$. The value of the expression is as follows:

$$
\left\{\begin{array}{l}
h_{1}=\arctan \frac{Y^{\prime}}{X^{\prime}}, \quad X^{\prime}>0, \\
h_{1}=180+\arctan \frac{Y^{\prime}}{X^{\prime}}, \quad X^{\prime}<0, \\
h_{1}=90^{\circ}, \quad X^{\prime}=0, Y^{\prime}>0, \\
h_{1}=-90^{\circ}, \quad X^{\prime}=0, Y^{\prime}<0, \\
h_{2}=\arcsin \frac{Z^{\prime}}{R^{\prime}} .
\end{array}\right.
$$

From (33), it can be seen that for any spatial position of the sound source, the horizontal declination angle and the elevation angle value can be obtained. In combination with (32), the omnidirectional positioning calibration of the sound source can be realized.

\section{The Simulation Results}

Based on the array of seven-element microphones, the sound source geometric localization algorithm was introduced into the model, the sound source azimuth was simulated on the basis of the forward data, the sound source data measured from the array observation angle was used for sound source localization calibration, and two experiments were performed: Experiment 1 is a simulative experiment of a omnidirectional localization algorithm based on a sevenelement microphone array, and Experiment 2 is a simulation experiment of an omnidirectional positioning calibration method for a sound source.

In Experiment 1, in order to verify the positioning accuracy of the sound source geometric positioning algorithm, a seven-element microphone array model was used to simulate it. According to Figures 1-4, the parameters are set: $c$ is equal to 340 meters per second, and $a$ is equal to 2 meters. The four forward datasets are as follows: $x$ equals 80 meters, $y$ equals -60 meters, and $z$ equals 120 meters; $x$ equals -50 meters, $y$ equals 0 meters, and $z$ equals -130 meters; $x$ equals 0 meters, $y$ equals $70 \mathrm{~m}$, and $z$ equals 150 meters; and $x$ equals 0 meters, $y$ equals 0 meters, and $z$ equals -80 meters. The omnidirectional localization simulation results of a sound source based on a seven-element microphone array are shown in Table 1.

From Table 1, it can be seen that although the simulation location of the sound source is different from the theoretical location, the error rate of the sound source coordinate parameters $x^{\prime}, y^{\prime}$, and $z^{\prime}$ is less than $0.01 \%$, which is still within the acceptable range; compared with the theoretical angle, the deviation of simulation is also smaller, and the angle error rate is about $0.005 \%$, which indicates that the sound source has a high geometric localization accuracy and has a very good sound source omnidirectional positioning effect.

In Experiment 2, using (32) and (33), the sound source was omnidirectionally positioned and calibrated to verify the accuracy of the calibration method. The basic parameters are set as follows: $c$ is equal to 340 meters per second, $a$ is equal to 2 meters, and two sets of forward data are selected (unit: meters): $(60,-40,80)$ and $(-50,0,-60)$. The set observation angle $N$ is (unit: $\mathrm{m}):(20,30,40)$, and the results of the omnidirectional positioning calibration of the sound source are shown in Table 2.

As can be seen from Table 2, the calibrated sound source position is close to the theoretical value. The sound source localization calibration is based on the sound source geometric localization algorithm. The calibrated sound source localization error rate is lower than $0.01 \%$, and the error rate of horizontal declination angle $h_{1}$ and elevation angle $h_{2}$ is less 
TABLE 1: Sound source omnidirectional localization simulation results based on seven-element microphone array.

\begin{tabular}{|c|c|c|c|c|c|}
\hline $\begin{array}{l}\text { Theory location } \\
\text { (meters) }\end{array}$ & $\begin{array}{l}\text { Theoretical angle } \\
\text { (degrees) }\end{array}$ & $\begin{array}{c}\text { Simulation location } \\
\text { (meters) }\end{array}$ & $\begin{array}{l}\text { Simulation angle } \\
\text { (degrees) }\end{array}$ & $\begin{array}{l}\text { Location error } \\
\text { rate }(\%)\end{array}$ & $\begin{array}{l}\text { Angle error } \\
\text { rate }(\%)\end{array}$ \\
\hline \multirow{2}{*}{$(80,-60,120)$} & $H_{1}=-36.8699$ & \multirow{2}{*}{$(79.9952,-59.9958,120.0053)$} & $H_{1}^{\prime}=-36.8696$ & \multirow{2}{*}{$(0.006,0.007,0.0044)$} & 0.0024 \\
\hline & $H_{2}=50.1944$ & & $H_{2}^{\prime}=50.1975$ & & 0.0052 \\
\hline \multirow{2}{*}{$(-50,0,-130)$} & $H_{1}=180$ & \multirow{2}{*}{$(-49.9955,0,-130.0017)$} & $H_{1}^{\prime}=180$ & \multirow{2}{*}{$(0.009,0,0.0013)$} & 0 \\
\hline & $\mathrm{H}_{2}=-68.9625$ & & $H_{2}{ }^{\prime}=-68.9645$ & & 0.0022 \\
\hline \multirow{2}{*}{$(0,70,150)$} & $H_{1}=90$ & \multirow{2}{*}{$(0,69.9958,150.0020)$} & $\mathrm{H}_{1}{ }^{\prime}=90$ & \multirow{2}{*}{$(0,0.006,0.0013)$} & 0 \\
\hline & $H_{2}=64.9831$ & & $H_{2}^{\prime}=64.9847$ & & 0.0014 \\
\hline \multirow{2}{*}{$(0,0,-80)$} & $H_{1}=0$ & \multirow{2}{*}{$(0,0,-80)$} & $H_{1}{ }^{\prime}=0$ & \multirow{2}{*}{$(0,0,0)$} & 0 \\
\hline & $H_{2}=-90$ & & $H_{2}{ }^{\prime}=-90$ & & 0 \\
\hline
\end{tabular}

TABLE 2: The simulation results of the sound source omnidirectional positioning calibration method.

\begin{tabular}{lccc}
\hline Theory location (meters) & Calibration theoretical data & Calibration simulation data & Error rate $(\%)$ \\
\hline & $(40,-70,40)$ & $(39.9929,-69.9941,40.0083)$ & $0.018,0.0084,0.021$ \\
$(60,-40,80)$ & $h_{1}=-60.2551$ & $h_{1}=-60.2574$ & 0.0038 \\
& $h_{2}=26.3878$ & $h_{2}=26.395$ & 0.0272 \\
\hline$(-50,0,-60)$ & $(-70,-30,-100)$ & $(-69.9903,-30,-100.008)$ & $0.0139,0,0.008$ \\
& $h_{1}=203.199$ & $h_{1}=203.201$ & 0.001 \\
& $h_{2}=-52.7079$ & $h_{2}=-52.7134$ & 0.0104 \\
\hline
\end{tabular}

than $0.005 \%$. The theoretical simulation data obtained is more accurate. It can give the observer accurate source location information and fully show the advantages of the omnidirectional positioning calibration method based on the seven-element microphone array.

\section{Actual Measurement Results}

In the actual measurement experiment, this article uses the acoustic sensor to construct the seven-element microphone array passive acoustic data acquisition system, write the program on the Keil4 software platform, use Flymcu to receive the serial port transmission data, and record the five relative time delay values displayed in the text interface, namely, $T_{1}$, $T_{2}, T_{3}, T_{4}$, and $T_{5}$. Use the measured relative delay values to calculate the calibrated sound source azimuth $\left(X^{\prime}, Y^{\prime}, Z^{\prime}\right)$, horizontal declination angle $h_{1}$, and elevation angle $h_{2}$. At different time points in April 2018, the indoor test sites were selected at the Nanjing University of Information Science \& Technology, Pukou District, Nanjing, using a portable Bluetooth stereo to simulate sound sources. Based on the coordinate system where the seven-element microphone array is located, with the microphone M0 as the origin, the position where the three-dimensional coordinates have been measured: $(-0.5 \mathrm{~m}, 2 \mathrm{~m}, 1.6 \mathrm{~m}),(1.8 \mathrm{~m}, 3.7 \mathrm{~m},-0.9 \mathrm{~m})$, and $(1.4 \mathrm{~m}, 2.3 \mathrm{~m}, 2.6 \mathrm{~m})$, emits sound source for actual test experiment. The actual sound source omnidirectional positioning calibration experiment diagram is shown in Figure 13. The observation angle $N$ is located at $(1.6 \mathrm{~m}, 2.7 \mathrm{~m}, 1 \mathrm{~m})$, corresponding to the three groups of sound source coordinates, and the actual array spacing $a$ is

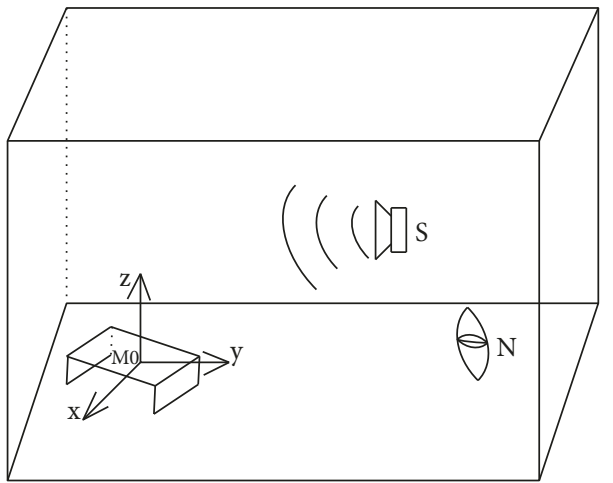

FIgURE 13: Actual sound source omnidirectional positioning calibration experiment.

$0.4 \mathrm{~m}, 0.6 \mathrm{~m}$, and $0.8 \mathrm{~m}$. The relative delay values, sound source localization, and angle calibration data are shown in Tables 3-6.

From Tables 3-6, it can be seen that when there is less noise and reverberation in the indoor environment, the measured calibration data based on the seven-element microphone array model and the error rate are in the controllable range although they are different from the theoretical data. The actual measured data is more reliable.

As can be seen from Tables 3-5, the actual measured horizontal declination angle $h_{1}$ and elevation angle $h_{2}$ are significantly different from the theoretical data. In combination with (7), the horizontal declination angle $H_{1}{ }^{\prime}$ is related to the sound source coordinate parameters $x^{\prime}$ and $y^{\prime}$; however, the value of elevation $\mathrm{H}_{2}{ }^{\prime}$ is not only related to the sound source 
TABle 3: The actual calibration data on April 2nd.

\begin{tabular}{lccc}
\hline$a=0.4 \mathrm{~m}$ & Localization (meters) & Horizontal declination (degrees) & Elevation angle (degrees) \\
\hline Actual calibration & $(-2.03958,-0.639739,0.544045)$ & 197.4150 & 14.2796 \\
Theoretical calibration & $(-2.09443,-0.709757,0.613837)$ & 198.7200 & 15.5133 \\
\hline
\end{tabular}

TABle 4: The actual calibration data on April 9th.

\begin{tabular}{lccc}
\hline$a=0.6 \mathrm{~m}$ & Localization (meters) & Horizontal declination (degrees) & Elevation angle (degrees) \\
\hline Actual calibration & $(0.160333,1.06489,-1.96708)$ & 81.4377 & -61.3012 \\
Theoretical calibration & $(0.185202,0.991324,-1.9629)$ & 79.4178 & -62.8074 \\
\hline
\end{tabular}

TABLe 5: The actual calibration data on April 12th.

\begin{tabular}{lccc}
\hline$a=0.8 \mathrm{~m}$ & Localization (meters) & Horizontal declination (degrees) & Elevation angle (degrees) \\
\hline Actual calibration & $(-0.245108,-0.383802,1.60774)$ & 237.4360 & 74.1852 \\
Theoretical calibration & $(-0.226879,-0.432551,1.64258)$ & 242.3220 & 73.4395 \\
\hline
\end{tabular}

TABLE 6: The error rate of the actual measured sound source omnidirectional positioning calibration data.

\begin{tabular}{lccc}
\hline Date & Coordinate error (\%) & Horizontal deflection error (\%) & Elevation angle error (\%) \\
\hline April 2nd & $(2.62,9.87,11.37)$ & 0.66 & 7.95 \\
April 9th & $(13.43,7.42,0.21)$ & 2.54 & 2.40 \\
April 12th & $(8.03,11.20,2.12)$ & 2.02 & 1.02 \\
\hline
\end{tabular}

coordinate parameter $x^{\prime}$ and the distance $r^{\prime}$ from the sound source to the center of the array but also related to $H_{1}{ }^{\prime}$. When there is an error in the measured sound source coordinate parameters, the horizontal declination angle $h_{1}$ and the elevation angle $h_{2}$ obtained after calibration must also have errors. Although there are some errors, compensation can be made by increasing the accuracy of the relative delay measurement.

From Table 6, it can be seen that with the increase of the actual spacing of the array elements, due to the existence of large sites and model volume factors, only the maximum array element spacing of 0.8 meters is taken, but the error rate of sound source coordinates, horizontal declination angle, and elevation angle has a tendency of getting lower and lower, the error rate of sound source coordinates is around $10 \%$, the horizontal declination angle error is less than $5 \%$, and the elevation angle error is less than $8 \%$, which is close to the theoretical data. Combined with the error analysis part of the text, if we further increase the array element spacing between 2 and 10 meters, we can more fully demonstrate the superiority of the sound source omnidirectional positioning calibration method based on microphone observation angle.

In summary, in the actual measurement, the sound source omnidirectional positioning calibration method based on microphone observation angle is close to the simulation effect. Through actual measurement experiments, it is proved that the actual calibration accuracy can be more ensured after appropriately increasing the array element spacing.

\section{Conclusions}

Based on the seven-element microphone array model, the sound source geometric localization algorithm was introduced into the array model, and the sound source data are measured by array angle. This paper proposes a sound source omnidirectional positioning calibration method based on microphone observation angle. By determining the polarity of the elevation angle, omnidirectional sound source positioning can be achieved. The errors caused by the array parameters are analyzed, and some improved methods are proposed. A new coordinate system is established. From the perspective of the observation point, then the location of the sound source is calibrated. Simulation and actual measurement experiments show that the method has high positioning accuracy and can obtain accurate calibration results.

The seven-element microphone array is arranged in a cross array on a three-dimensional coordinate axis, and the array element spacing between the microphones can be adjusted to reduce the array element density in the actual arrangement area. Appropriately increasing the array element spacing can also improve the accuracy of the actual sound source omnidirectional positioning calibration. Four kinds of array models were established to solve the problem of calculating blind spots in the sound source position in the actual environment. Based on the model, the derivation of the relevant formulas for the omnidirectional positioning calibration of the sound source is carried 
out, and the methods were applied to actual measurement experiments to make the sound source positioning calibration more comprehensive.

Of course, there is still much room for improvement in the accuracy of the calibration of sound source azimuths based on the array angle. In a real complex environment, microphones should be placed reasonably. The relative time delay value will still be affected by attenuation and outside interference during sound source propagation; there are certain errors that affect sound source positioning calibration. There are many problems involved. Assuming that the model is also ideal, how to transfer the actual indoor measurement experiment to the field for performance testing in a complex real environment requires further exploration and research in the following work.

\section{Data Availability}

The data used to support the findings of this study are available from the corresponding author upon request.

\section{Conflicts of Interest}

The authors declare that there are no conflicts of interest regarding the publication of this paper.

\section{Acknowledgments}

This work is supported by the National Natural Science Foundation of China (Grant no. 61671248 and no. 41605121), the Key Research and Development Plan of Jiangsu Province, China (Grant no. BE2018719), and the Advantage Discipline "Information and Communication Engineering" of Jiangsu Province, China.

\section{References}

[1] F. Asp, A.-M. Jakobsson, and E. Berninger, "The effect of simulated unilateral hearing loss on horizontal sound localization accuracy and recognition of speech in spatially separate competing speech," Hearing Research, vol. 357, pp. 54-63, 2018.

[2] S. Shahnawazuddin and R. Sinha, "Sparse coding over redundant dictionaries for fast adaptation of speech recognition system," Computer Speech \& Language, vol. 43, pp. 1-17, 2017.

[3] X. Zhou, X. Liu, C. Yang, A. Jiang, and B. Yan, "Multi-channel features spatio-temporal context learning for visual tracking," IEEE Access, vol. 5, pp. 12856-12864, 2017.

[4] L. Wei, M. Li, S. Qin, Q. Fu, and D. Yang, "Sound source localization method in an environment with flow based on AmietIMACS," Mechanical Systems and Signal Processing, vol. 88, pp. 240-252, 2017.

[5] X. Zhong, L. Sun, and W. Yost, "Active binaural localization of multiple sound sources," Robotics and Autonomous Systems, vol. 85, pp. 83-92, 2016.

[6] X.-Z. Zhang, C.-X. Bi, Y.-B. Zhang, and L. Xu, "A time-domain inverse technique for the localization and quantification of rotating sound sources," Mechanical Systems and Signal Processing, vol. 90, pp. 15-29, 2017.

[7] C. Yang, K. Huang, H. Cheng, Y. Li, and C.-Y. Su, "Haptic identification by ELM-controlled uncertain manipulator,"
IEEE Transactions on Systems, Man, and Cybernetics: Systems, vol. 47, no. 8, pp. 2398-2409, 2017.

[8] C. Yang, J. Luo, Y. Pan, Z. Liu, and C.-Y. Su, "Personalized variable gain control with tremor attenuation for robot teleoperation," IEEE Transactions on Systems, Man, and Cybernetics: Systems, vol. 48, no. 99, pp. 1-12, 2017.

[9] Q. Zhu, H. Li, R. Ye, M. Xu, and F. Zheng, "Development of alarm system based on speech recognition," in Proceedings of the 2016 7th International Conference on Education, Management, Computer and Medicine (EMCM 2016), Shenyang, China, February 2017.

[10] A. V. Oppenheim and J. S. Lim, “The importance of phase in signals," Proceedings of the IEEE, vol. 69, no. 5, pp. 529-541, 2005.

[11] A. V. Oppenheim, J. S. Lim, and S. R. Curtis, "Signal synthesis and reconstruction from partial Fourier-domain information," Journal of the Optical Society of America, vol. 73, no. 11, pp. 1413-1420, 1983.

[12] A. Oppenheim, J. Lim, G. Kopec et al., "Phase in speech and pictures," in ICASSP '79. IEEE International Conference on Acoustics, Speech, and Signal Processing, pp. 632-637, Washington, DC, USA, April 1979.

[13] L. Liu, J. He, and G. Palm, "Effects of phase on the perception of intervocalic stop consonants," Speech Communication, vol. 22, no. 4, pp. 403-417, 1997.

[14] F. Yu, D. Li, Q. Guo, B. Zeng, and Z. Wang, "The performance analysis for distributed collaborative beamforming transmission of clustered mobile sensor network," Journal of Harbin Institute of Technology, vol. 23, no. 4, pp. 8-14, 2016.

[15] M. Aldeman and G. Raman, "Effects of array scaling and advanced beamforming algorithms on the angular resolution of microphone array systems," Applied Acoustics, vol. 132, pp. 58-81, 2018.

[16] J. S. Lim and F. U. Dowla, "A new algorithm for highresolution two-dimensional spectral estimation," Proceedings of the IEEE, vol. 71, no. 2, pp. 284-285, 1983.

[17] X. Zhou, X. Liu, A. Jiang, B. Yan, and C. Yang, "Improving video segmentation by fusing depth cues and the visual background extractor (ViBe) algorithm," Sensors, vol. 17, no. 5, article 1177, 2017.

[18] L. Huang, S. Wu, D. Feng, and L. Zhang, "Low complexity method for signal subspace fitting," Electronics Letters, vol. 40, no. 14, pp. 847-848, 2004.

[19] K. K. Paliwal and L. D. Alsteris, "On the usefulness of STFT phase spectrum in human listening tests," Speech Communication, vol. 45, no. 2, pp. 153-170, 2005.

[20] L. D. Alsteris and K. K. Paliwal, "Short-time phase spectrum in speech processing: a review and some experimental results," Digital Signal Processing, vol. 17, no. 3, pp. 578616, 2007.

[21] L. D. Alsteris and K. K. Paliwal, "Further intelligibility results from human listening tests using the short-time phase spectrum," Speech Communication, vol. 48, no. 6, pp. 727-736, 2006.

[22] K. Paliwal, K. Wojcicki, and B. Shannon, "The importance of phase in speech enhancement," Speech Communication, vol. 53, no. 4, pp. 465-494, 2011.

[23] M. S. Brandstein and D. B. Ward, "Microphone arrays: signal processing techniques and applications," in IEEE Transactions on Acoustics, Speech and Signal Processing, 2001, ICASSP-88. 
[24] V. Kudriashov, "Improvement of range estimation with microphone array," Cybernetics and Information Technologies, vol. 17, no. 1, pp. 113-125, 2017.

[25] J. Li, L. Wang, and J. Y. Meng, "Simulation research on structure-changing control over the follow-up system of the smart mine," Journal of Beijing Institute of Technology(English Edition), vol. 9, pp. 278-282, 2000.

[26] T. L. Ju, Q. C. Peng, and H. Z. Shao, "Research of speech source localizations sub-array algorithms in near field based on microphone arrays," Journal of Electronic Measurement and Instrument, vol. 20, pp. 50-55, 2006.

[27] T. L. Ju, H. Z. Shao, and Q. Z. Peng, "Speech source 3D localization MUSIC algorithm in near field," Journal of Electronic Measurement and Instrument, vol. 21, pp. 44-48, 2007.

[28] Y. Ding, Q. Li, W. Liao, R. Zeng, and Z. Su, "Switching impluse discharge characteristics and altitude corrections for typical long air gaps at high altitude areas," High Voltage Engineering, vol. 39, pp. 1441-1446, 2013.

[29] H. Y. Xing, Q. Zhang, W. Xu, and X. Y. Ji, "Altitude correction method and networking for atmospheric electric field data," Journal of PLA University of Science and Technology(Natural Science Edition), vol. 15, pp. 591-597, 2014.

[30] B. H. Wang, Y. L. Wang, H. Chen, and Y. Guo, "The auxiliary element method for azimuth dependent array phase error correction," Science in China, Ser. E, vol. 34, pp. 906-918, 2004.

[31] C. Yang, X. Wang, Z. Li, Y. Li, and C.-Y. Su, "Teleoperation control based on combination of wave variable and neural networks," IEEE Transactions on Systems, Man, and Cybernetics: Systems, vol. 47, no. 8, pp. 2125-2136, 2017.

[32] M. Coutino, M. B. Møller, J. K. Nielsen, and R. Heusdens, "Greedy alternative for room geometry estimation from acoustic echoes: a subspace-based method," in 2017 IEEE International Conference on Acoustics, Speech and Signal Processing (ICASSP), New Orleans, LA, USA, March 2017.

[33] D. Ozevin, "Geometry-based spatial acoustic source location for spaced structures," Structural Health Monitoring: An International Journal, vol. 10, no. 5, pp. 503-510, 2011.

[34] F. Joublin and T. Rodemann, "Method for estimating the position of a sound source for online calibration of auditory cue to location transformations," US Patent 8036397B2, 2011.

[35] F. Martellotta, "On the use of microphone arrays to visualize spatial sound field information," Applied Acoustics, vol. 74, no. 8, pp. 987-1000, 2013.

[36] D. A. Dick and M. C. Vigeant, "A comparison of measured room acoustics metrics using a spherical microphone array and conventional methods," Applied Acoustics, vol. 107, pp. 34-45, 2016.

[37] C. Yang, Y. Jiang, Z. Li, W. He, and C.-Y. Su, "Neural control of bimanual robots with guaranteed global stability and motion precision," IEEE Transactions on Industrial Informatics, vol. 13, no. 3, pp. 1162-1171, 2017.

[38] B. Rafaely, "Analysis and design of spherical microphone arrays," IEEE Transactions on Speech and Audio Processing, vol. 13, no. 1, pp. 135-143, 2005.

[39] H. Yilmaz and H. S. Sazak, "Double-looped maximum likelihood estimation for the parameters of the generalized gamma distribution," Mathematics and Computers in Simulation, vol. 98, no. 2, pp. 18-30, 2014.
[40] C. Yang, C. Zeng, P. Liang, Z. Li, R. Li, and C.-Y. Su, "Interface design of a physical human robot interaction system for human impedance adaptive skill transfer," IEEE Transactions on Automation Science and Engineering, vol. 15, no. 1, pp. 329-340, 2018.

[41] D. Q. Wang, Z. Zhang, and J. Y. Yuan, "Maximum likelihood estimation method for dual-rate Hammerstein systems," International Journal of Control Automation and Systems, vol. 15, no. 2, pp. 698-705, 2017. 


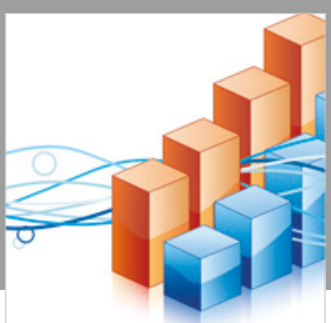

Advances in

Operations Research

\section{-n-m}
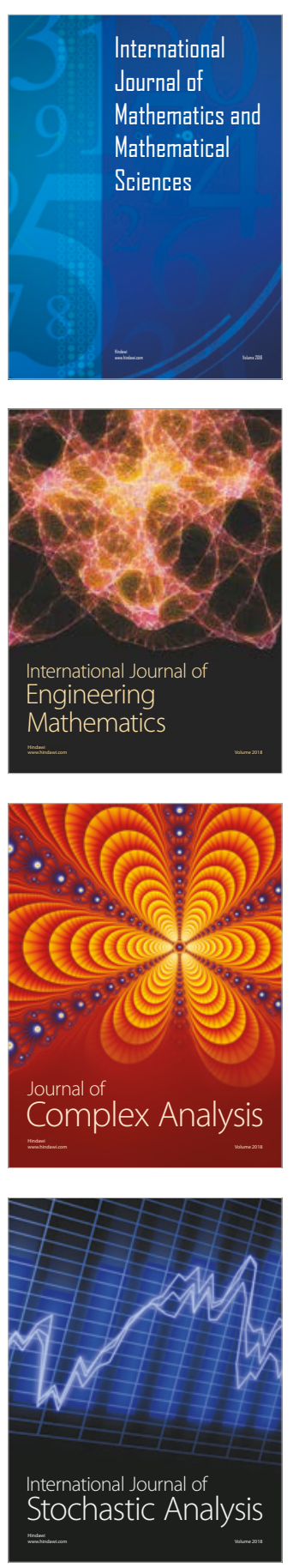
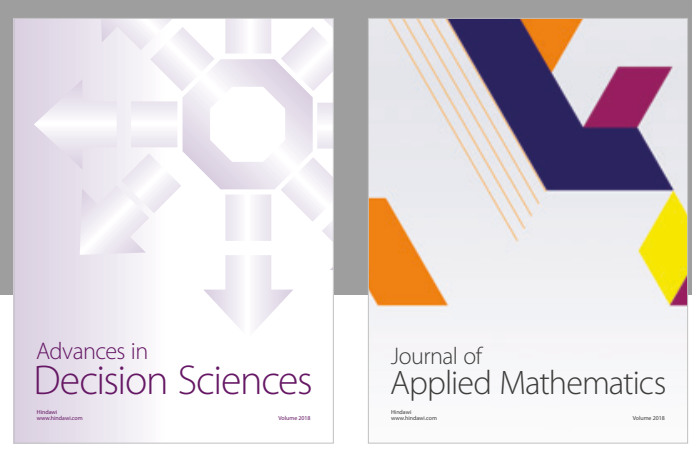

Journal of

Applied Mathematics
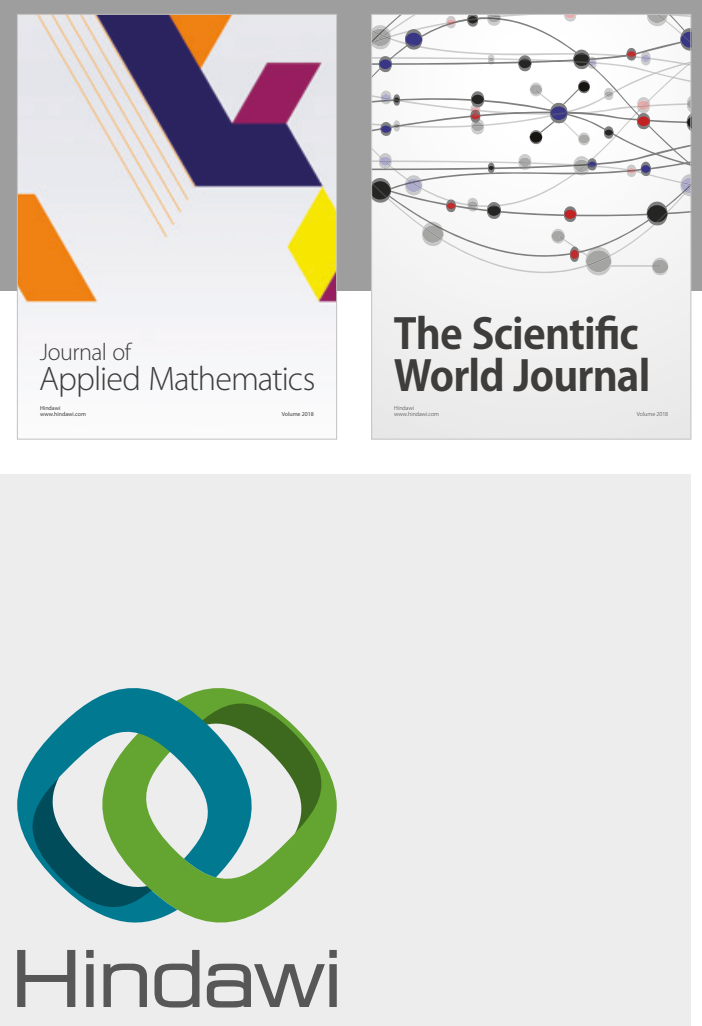

Submit your manuscripts at

www.hindawi.com

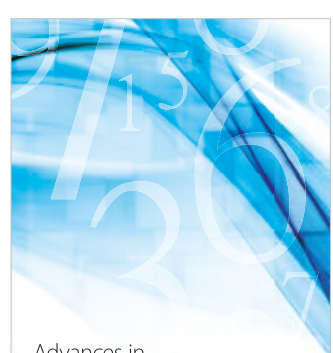

Advances in
Numerical Analysis
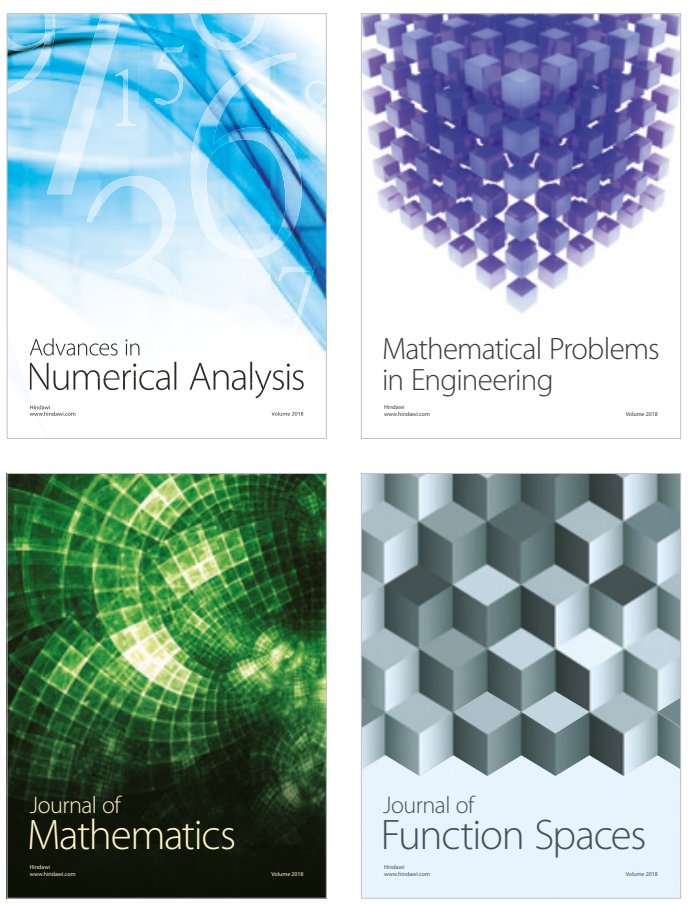

Mathematical Problems in Engineering

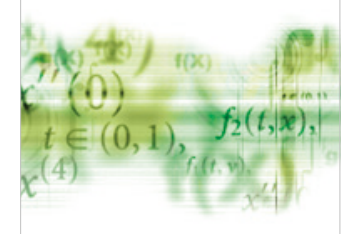

International Journal of

Differential Equations

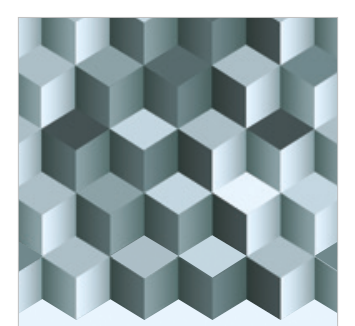

Journal of

Function Spaces
The Scientific

World Journal

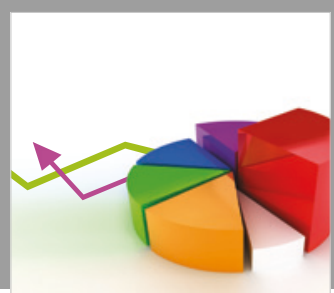

Journal of

Probability and Statistics
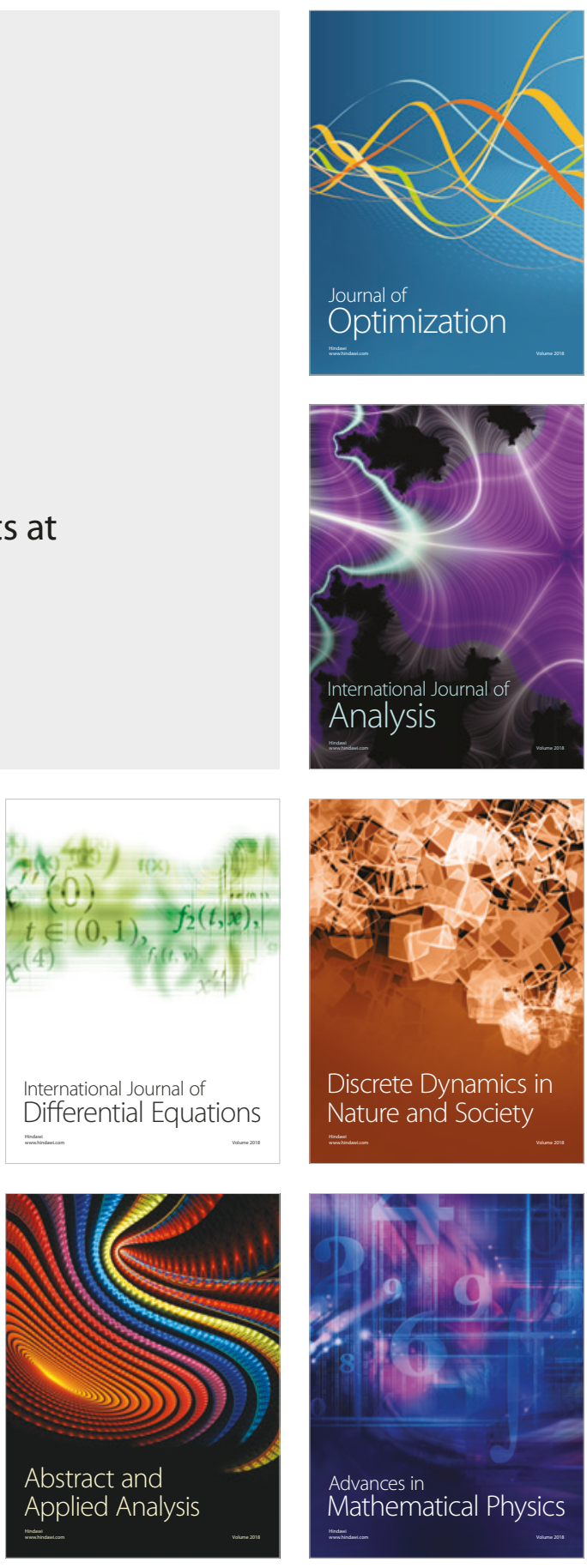